\title{
Photoluminescence of erbium doped microcrystalline silicon thin films produced by reactive magnetron sputtering
}

\author{
M. F. Cerqueira* ${ }^{1}$, M. V. Stepikhova ${ }^{2}$ and J. A. Ferreira ${ }^{1}$ \\ ${ }^{1}$ Physics Department, University of Minho, Largo do Paço 4700-320 Braga Portugal \\ ${ }^{2}$ Institute for Physics of Microstructures RAS, 603600 Nizhnij Novgorod GSP-105 Russia
}

\begin{abstract}
Microcrystalline silicon thin films doped with erbium were produced by RF sputtering and their structural, chemical and optical properties were studied by X-ray diffractometry at grazing incidence, Rutherford back scattering and optical transmission spectroscopy. The samples exhibit a sharp photoluminescence (PL) spectrum from the Er centres with the strongest peak positioned at $1.536 \mu \mathrm{m}$ with a full width at half maximum of about $8 \mathrm{~nm}$. When the temperature varies between $5 \mathrm{~K}$ and $300 \mathrm{~K}$ the photoluminescence decreases only five fold, in contrast to the behaviour reported for monocrystalline silicon.
\end{abstract}

Keywords: Photoluminescence, Erbium, nanocrystalline silicon

\section{Introduction}

During the last 10 years, modern growth techniques have made possible the fabrication of semiconductor nano-structures of typical size $<10 \mathrm{~nm}$, with spatial confinement of electrons in all three dimensions. These systems are of considerable current interest because of the new physics involved and potential device applications.

One of these applications relates to Si-based optoelectronics. Erbium doped silicon is a recognised candidate to become a material for emitters and detectors to be used in optical communication systems. However, there are problems with efficient energy transfer from the Si matrix to the Er centres at room temperature. The use of Er-doped Si nano-crystallites can help to overcome these problems [1]. Studies of the photoluminescence of Er-doped nanocrystalline silicon constitute an important probe for these applications.

\section{Experimental}

Erbium doped microcrystalline silicon thin films were grown by reactive magnetron sputtering on ordinary glass substrates using a procedure similar to that used for the preparation of undoped $\mu \mathrm{c}-\mathrm{Si}: \mathrm{H}$

*Corresponding author: tel: +253 604332; fax: +253678981; e-mail: fcerqueira@ fisica.uminho.pt films [2], but adding small pieces of metallic erbium to the c-Si target. The target used was a c-Si with high purity $(99.99 \%)$. The erbium was placed in a low erosion area of the silicon target, in order to 
obtain low concentrations. The substrate target distance was fixed at $55 \mathrm{~mm}$. Two series of samples were grown, for different amounts of erbium on the c-Si target. In each series we have changed basically the substrate temperature since it is this parameter that control the crystal grain size [3]. Growth conditions are presented in Table 1. The first series (Er1,2 and 3) was grown in a argon rich atmosphere and the second series (Er4,5 and 6) in a hydrogen rich atmosphere.

Table 1

Growth conditions

\begin{tabular}{|c|c|c|c|c|}
\hline Sample & $\begin{array}{c}\text { Temperature } \\
\left({ }^{\circ} \mathrm{C}\right)\end{array}$ & $\begin{array}{c}\mathrm{RF} \text { power } \\
(\mathrm{W})\end{array}$ & $\mathrm{H}_{2}\left(\mathrm{~cm}^{3} / \mathrm{min}\right)$ & $\begin{array}{c}\mathrm{Ar} \\
\left(\mathrm{cm}^{3} / \mathrm{min}\right)\end{array}$ \\
\hline Er1 & 250 & 40 & 20 & 100 \\
\hline Er2 & 350 & 40 & 20 & 100 \\
\hline Er3 & 100 & 40 & 20 & 100 \\
\hline Er4 & 250 & 80 & 50 & 30 \\
\hline Er5 & 350 & 80 & 50 & 30 \\
\hline Er6 & 100 & 80 & 50 & 30 \\
\hline
\end{tabular}

For the structural characterisation we used X-ray diffractometry at the grazing incidence geometry. The voltage was $40 \mathrm{kV}(35 \mathrm{~mA})$, the incidence angle was fixed at $0.5^{\circ}$, the step was $0.04^{\circ}$ and the acquisition time by step was $5.0 \mathrm{~s}$. The range studied was from 23 to $71^{\circ}$. The excitation used was the Cobalt line with $\lambda=1.78896 \breve{A}$. The chemical composition was determined by Rutherford Back Scattering (RBS) technique. The thickness was evaluated from the analysis of the near I.R. transmission spectra of the films. The Photoluminescence study was done with a high resolution Fourier set-up (BOMEM spectrometer DA8) using the $514.5 \mathrm{~nm}$ line of an argon laser for excitation.

\section{Results and discussion}

\subsection{Structural and chemical characterisation}

From the interference pattern of the transmission spectra the thicknesses of the films were obtained using the Swanepoel method [4]. Comparing the transmission spectra we verify that the samples grown in a rich argon (Ar) atmosphere show a larger number of interference fringes, compared with the samples grown in a rich hydrogen $\left(\mathrm{H}_{2}\right)$ atmosphere, indicating that the thickness of those films is higher. This is due to the high sputtering efficiency of the argon atoms compared with the hydrogen atoms.

The chemical composition (see Table 2) was determined using the Rutherford Back Scattering technique. The percentage of Er and Si obtained from RBS is very accurate, but the values for oxygen are only approximate. It is nevertheless clear that for the samples grown in a $\mathrm{H}_{2}$ rich atmosphere and high temperatures (Er4 and Er5), the percentage of Si and $\mathrm{O}$ are comparable. We know from previous studies by ellipsometry that these nanostructured samples have a $\mathrm{SiO}$ top layer of approximately 60$100 \mathrm{~nm}$. For the same initial amount of Er in the sputtering target, the concentration of the dopant in the films is highest for the lowest substrate temperature.

Our samples have also been studied at room temperature by X-ray diffractometry at grazing incidence. The samples were annealed at $500^{\circ} \mathrm{C}$ for 30 minutes in $\mathrm{N}_{2}$ atmosphere. The X-ray experiments were done on the annealed and as-grown samples. All the samples show the broad band related to the silicon amorphous matrix. The as-grown samples do not exhibit any structure. The (111) diffraction peak of c- Si is only visible, albeit with low intensity, in the Er5 annealed sample. The diffraction peak analysis, by fitting a pseudo-Voigt function [2] to the (111) c-Si diffraction peak gives an average 
crystal size of $270 \breve{A}$. Previous studies have shown that the band gap for crystals of this size is higher than $1.9 \mathrm{eV}$.

Table 2

Atomic concentration and thickness for different samples

\begin{tabular}{|c|c|c|c|c|c|}
\hline Sample & $\begin{array}{c}\text { Er } \\
(\%)\end{array}$ & $\begin{array}{c}\mathrm{Si} \\
(\%)\end{array}$ & $\begin{array}{c}\text { O } \\
(\%)\end{array}$ & $\begin{array}{c}\text { Ar } \\
(\%)\end{array}$ & $\begin{array}{c}\text { Thickness } \\
(\mathrm{nm})\end{array}$ \\
\hline Er1 & 4.0 & 76 & 19 & 1 & 722 \\
\hline Er2 & 4.1 & 79 & 16 & 1 & 777 \\
\hline Er3 & 4.7 & 78 & 16.3 & - & 786 \\
\hline Er4 & 0.3 & 50.7 & 49 & - & 270 \\
\hline Er5 & 0.3 & 42.7 & 57 & - & $<200$ \\
\hline Er6 & 0.7 & 77 & 22 & - & 407 \\
\hline
\end{tabular}

\subsection{Photoluminescence study}

The photoluminescence of these films was measured using the excitation wavelength $514.5 \mathrm{~nm}$ of Ar laser. The laser power was $200 \mathrm{~mW}$ and the measurement temperature was $5 \mathrm{~K}$, except for the temperature dependence study.

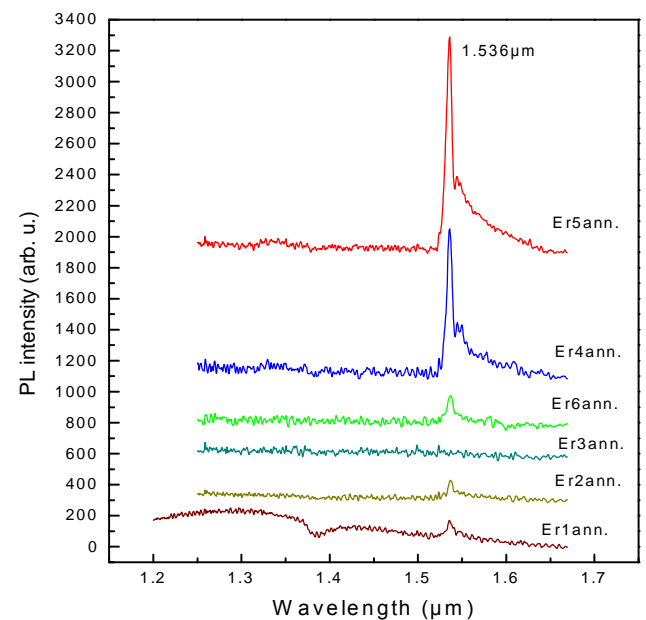

a)

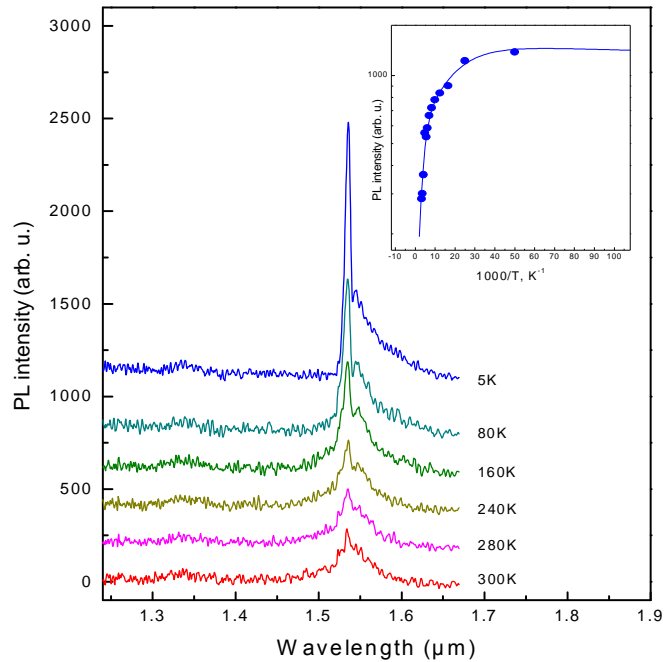

b)

Figure 1 - a) Photoluminescence for the annealed Erbium doped microcrystalline sample. b) Photoluminescence behaviour with temperature for Er5 annealed sample.

Figure 1a) shows the photoluminescence spectrum of the annealed samples. These spectra are characterised by a peak around $1.536 \mu \mathrm{m}$. This peak is the signature of the erbium centres. The PL intensity of each sample increases by several orders of magnitude if the sample is annealed, especially in the case of Er5 sample. The increase of PL after annealing could be due the formation of Si nanocrystals during the annealing, to the annealing of some structure defects or to the decrease of hydrogen or oxygen concentration during the annealing process. According to the X-ray study we think that the first possibility is indeed true for Er5, which means that with the annealing we promote the crystallisation of some silicon, although for the other samples this is not the case.

In Fig. 1b) we present the result of the photoluminescence temperature study for the Er5 annealed sample. Increasing the temperature, from $5 \mathrm{~K}$ to $300 \mathrm{~K}$, the PL intensity decreases only 5 times. This 
temperature effect is attributed to thermally activated back transfer to the host and is less pronounced than in monocrystalline silicon [5]. This kind of behaviour is promising for applications but the interpretation of the PL origin is difficult. There exist two possibilities, concerning the location of the Er centres, namely the Er ions could be in the amorphous matrix or in the nanocrystals. Since all the samples (except Er3) show photoluminescence and only in the Er5 annealed sample there is evidence of the presence of nanocrystals, it appears that the Er centres are located in the a-Si:H matrix for Er1,2,4,6 samples.

The fact that PL quenching in all the samples studied, be lower than in monocrystalline silicon can be explained by the higher energy band gap [5,6] of the amorphous silicon (around $1.6 \mathrm{eV}$ for sputtered or evaporated material and for a typical hydrogen content - 20 at\%) compared with $1.1 \mathrm{eV}$ for c-Si. The back transfer process will then take place only at higher temperatures. This effect is even more remarkable for the nanocrystalline sample, where the energy gap is higher and where some Er can be located in the nanocrystalline phase.

Figure 2 shows the correlation between the photoluminescence intensity and the silicon hydrogen ratio content in the samples. It is visible from the

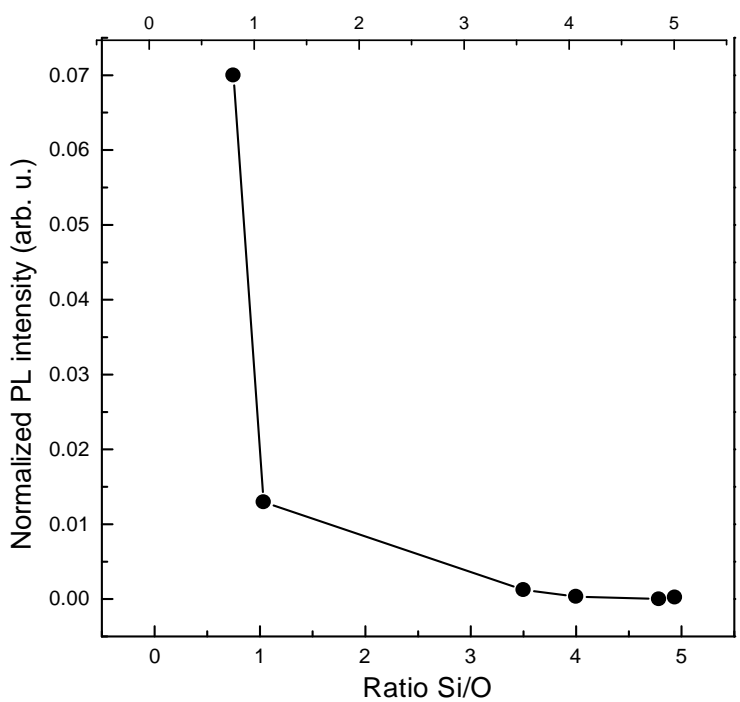

Figure 2- Normalised photoluminescence as a function of $\mathrm{Si} / \mathrm{O}$ content ration of the samples. figure that the presence of oxygen is fundamental for the PL intensity, as was already shown by others authors $[7,8]$.

\section{Conclusions}

The photoluminescence of Er incorporated in thin solid films of amorphous/nanocrystalline silicon was studied as a function of temperature and for different growing conditions. Results show that the Er ions are excited very efficiently through energy transfer from the host. The PL intensity of the sample having Si nanocrystals is much higher than for the others. The effect of increasing temperature on luminescence quenching is much less important than in monocrystalline silicon, and PL is still observed at room temperature. The PL intensity increases with $\mathrm{O} / \mathrm{Si}$ ratio of the films.

\section{References}

[1] - Y. Kanzawa, T. Kageyama, S. Takevka, M. Fijii, S. Hayashi, K. Yamamoto, Solid State commun. 102, 533 (1997)

[2] - M. F. Cerqueira, M. Andritschky, L. Rebouta, J. A. Ferreira and M. F. da Silva, Vacuum 46, 1385 (1995)

[3] - M. F. Cerqueira, J. A. Ferreira, M. Andritschky, Manuel F. M. Costa, Microelectronic Engineering 43-44, 627 (1998)

[4] - R. Swanepoel, J. Phys. E 16, 1214 (1983)

[5] - H. Przybylinska, W. Jantsch, Yu Suprun-Belevitch, M.Stepikhova, L. Palmetshofer, G. Hendorfer, A. Kozanecki, L. Palmetshofer, G. Hendorfer, A. Kozanecki, R. J. Wilson, B. J. Sealy, Phys. Rev. B 54, 2532 (1996)

[6] - A. Polman, J. Appl. Phys. 82, 1 (1997)

[7] - F. Priolo, S. Coffa, G. Franzo, C. Spinella, A. Carnera, V. Bellani, J. Appl. Phys. 74, 4936 (1993)

[8] - D. C. Adler, D. C. Jacobson, D. J. Eaglesham, M. A. Marcus, J. L. Benton, J. M. Poate, P. H. Citrin, Appl. Phys. Lett. 61,2181, (1992) 
Create PDF files without this message by purchasing novaPDF printer (http://www.novapdf.com) 